\title{
Author's reply: MRI in T staging of rectal cancer: How effective is it?
}

Dear Sir,

We thank the authors Gupta and Gupta ${ }^{[1]}$ for reading our article with keen interest. In response to the question, we have a similar cut off measurement at our institute. We also use " $5 \mathrm{~mm}$ " as the cut off on MRI for predicting CRM involvement. The CRM is reported as 'clear' or 'not under threat' if the tumour is beyond $5 \mathrm{~mm}$ and 'involved' if the tumour is within this distance. We specifically mention this in all our reports with radiological staging.
Mubashir Mulla

King's College Hospital, London, United Kingdom. E-mail: drbashir@rediffmail.com

\section{Reference}

1. Gupta M, Gupta R. MRI criterion for prediction of involvement of circumferential resection margin in rectal cancer. Indian J Radiol Imaging 2011;21:69-70. 